\title{
ON GALOIS REPRESENTATIONS VIA SIEGEL MODULAR FORMS OF GENUS TWO
}

\author{
MICHAEL DETTWEILER ${ }^{\dagger}$, ULF KÜHN, AND STEFAN REITER
}

\begin{abstract}
We study 4-dimensional Galois representations attached to Siegel modular forms. In some cases we determine the images of the absolute Galois group. This yields Galois realizations over $\mathbb{Q}$ for projective symplectic groups.
\end{abstract}

\section{Introduction}

It is well known that to classical modular forms there are attached twodimensional $l$-adic representations of the absolute Galois group $\mathrm{G}_{\mathbb{Q}}:=\operatorname{Gal}(\overline{\mathbb{Q}} / \mathbb{Q})$, see Deligne [6]. Ribet [15] has studied the images of $\mathrm{G}_{\mathbb{Q}}$ and proved that they are almost always "as big as possible". In particular, by considering modular forms of weight 24, he showed that the groups $\operatorname{PSl}\left(2, l^{2}\right)$ occur as Galois groups over $\mathbb{Q}$ if $l \neq 47$ and 144169 is a quadratic non-residue modulo $l$. Reverter and Vila [14] subsequently realized many other groups $P G l\left(2, l^{2 m-1}\right)$ and $P S l\left(2, l^{2 m}\right)$ as Galois groups over $\mathbb{Q}$ by considering modular forms of higher weights.

Results of Weissauer [24] associate four-dimensional Galois representations to Siegel modular forms of genus two (more generally, to any irreducible cuspidal automorphic representation of $G S p(4, \mathbb{A}), \mathbb{A}$ being the ring of adeles of $\mathbb{Q}$, whose component at infinity belongs to the holomorphic discrete series, see Theorem 4). We study the Galois representations attached to the unique (up to normalization and Galois conjugation) Siegel modular eigenform $\Upsilon$ of weight 28 on the full Siegel modular group $S p(4, \mathbb{Z})$ which is a cusp form and does not lie in the Maass Spezialschar. The first Fourier coefficients of $\Upsilon$ were determined by Skoruppa [18], [19].

The case of weight 28 is the smallest one where non-Maass, non-Eisenstein eigenforms exist whose Fourier coefficients do not lie completely in $\mathbb{Q}$ (see [18]) - so there is the chance of finding new Galois groups (see Remark 14 for details).

We use the following method to determine the image of $\mathrm{G}_{\mathbb{Q}}$ : We consider the image modulo $\lambda$, where $\lambda$ is a prime of some number field. Then we use the explicitly given characteristic polynomials of the images of the Frobenius elements, combining the results of Weissauer and Skoruppa, to find semisimple

Received January 2, 2001.

The first and the third author want to thank the Deutsche Forschungsgemeinschaft for financial support. 
elements in the image which do not fit simultaneously into any smaller subgroup than $S p\left(4, l^{n}\right)$.

We show that infinitely many of the groups $P S p\left(4, l^{2}\right)$ and $P G S p\left(4, l^{\prime 3}\right)$ (especially $P S p\left(4,19^{2}\right)$ and $P G S p\left(4,53^{3}\right)$ ) occur as Galois groups over $\mathbb{Q}$, see Thm $13 \mathrm{i})$ and ii). The corresponding Galois extensions are unramified outside $l$ and $l^{\prime}$, resp.. An argument similar to a result of Serre-Ribet, see Lemma 2, then implies that the corresponding $\lambda$-adic inverse images also occur as Galois groups over $\mathbb{Q}$.

We thank Professor Weissauer for providing us with his preprints and for valuable discussions. Further we thank J. Hartmann, J. Klüners, Professor Kramer and Professor Matzat for helpful comments.

\section{Lifting modular representations and reducing $l$-adic representations}

In this section we collect some general results about $l$-adic representations which will be useful later.

Notation 1. We denote by $\bar{K}$ an algebraic closure of a field $K$. By $\mathbb{P}$ we denote the set of primes of $\mathbb{N}$. If $p \in \mathbb{P}$, we denote by Frob $_{p}$ a Frobenius element of the absolute Galois group $\mathrm{G}_{\mathbb{Q}}:=\operatorname{Gal}(\overline{\mathbb{Q}} / \mathbb{Q})$, see Serre [16]. If $E$ is a number field and $\lambda$ is a place of $E$, we denote by $E_{\lambda}$ the completion of $E$ with respect to $\lambda$. If $K$ is a local field then we denote by $O_{K}$ (resp. $m_{K}, k_{K}$ ) its ring of integers (resp. the maximal ideal of $O_{K}$, its residue field).

Lemma 2. Let $K$ be a finite extension of $\mathbb{Q}_{l}, l \geq 5$. Suppose that $\mathcal{G}$ is a closed subgroup of $G S p\left(n, O_{K}\right)$ whose image "mod l" contains $S p\left(n, O_{K} / l O_{K}\right)$. Then $\mathcal{G}$ contains the group $\operatorname{Sp}\left(n, O_{K}\right)$.

Proof. This is analogous to the proof of Thm. 2.1 of Ribet [15]. The argument of Serre (as given in [15], p. 250) applies, if we can show that the Lie algebra $s p(n, q)$ "mod $\lambda$ " of $S p(n, q)$ is generated by nilpotent elements of degree two (here $\lambda$ denotes an extension of $l$ to $K$ such that $k_{K}=\mathbb{F}_{q}$ ):

By the Cartan decomposition, see [3], we have

$$
s p(n, q)=\mathfrak{t} \oplus \sum_{\alpha \in \Omega} \mathfrak{x}_{\alpha},
$$

where $\mathfrak{t}$ is the Lie algebra of a maximally split torus $T, \Omega$ denotes the root system and $\mathfrak{x}_{\alpha}$ is the root space of $\alpha($ "mod $\lambda ")$. Any element of $\mathfrak{x}_{\alpha}, \alpha \in \Omega$, is nilpotent of degree two. Thus it suffices to prove the claim for the elements of $\mathfrak{t}$, which are of the form $\operatorname{diag}(D,-D)$, where $D \in \operatorname{Mat}_{n / 2}(q)$ is a diagonal matrix. These elements can be written as

$$
\left(\begin{array}{cc}
D & 0 \\
0 & -D
\end{array}\right)=1 / 2\left(\begin{array}{cc}
D & D \\
-D & -D
\end{array}\right)+1 / 2\left(\begin{array}{cc}
D & -D \\
D & -D
\end{array}\right)
$$

where the summands on the right hand side lie in $s p(n, q)$ and are nilpotent of degree two. 
Lemma 3. Let $\tilde{K}$ be a finite extension of $\mathbb{Q}_{l}$ and $\rho: \mathrm{G}_{\mathbb{Q}} \mapsto G l(n, \tilde{K})$ be a continuous representation which is unramified outside a finite set $S \subseteq \mathbb{P}$ of primes. Let $K \subseteq \tilde{K}$ be the field which is generated over $\mathbb{Q}_{l}$ by the coefficients of the characteristic polynomials of all $\rho\left(\right.$ Frob $\left._{p}\right), p \in \mathbb{P} \backslash S$. Let further

$$
{ }^{-}: O_{\tilde{K}} \longrightarrow k_{\tilde{K}} \subseteq \overline{\mathbb{F}}_{l}
$$

denote the reduction modulo $m_{\tilde{K}}$. Set $\mathbb{F}_{q}=k_{K} \subseteq k_{\tilde{K}}$. Then there exists a representation

$$
\hat{\rho}: \mathrm{G}_{\mathbb{Q}} \longrightarrow G l\left(n, \mathbb{F}_{q}\right),
$$

such that $\rho$ factors through it and

$$
\overline{\operatorname{det}\left(1-X \cdot \rho\left(\operatorname{Frob}_{p}\right)\right)}=\operatorname{det}\left(1-\bar{X} \cdot \hat{\rho}\left(\operatorname{Frob}_{p}\right)\right) .
$$

Proof. By compactness, we can assume that the image of $\rho$ is contained in $G l\left(n, O_{\tilde{K}}\right)$. Let $\bar{\rho}: \mathrm{G}_{\mathbb{Q}} \rightarrow G l\left(n, \overline{\mathbb{F}}_{l}\right)$ be the composition of $\rho$ with the reduction modulo $m_{\tilde{K}}$. Let $\bar{\rho}_{s s}$ be the semisimplification of $\bar{\rho}$ in the sense of Serre [16], I-10. The character $\operatorname{tr}\left(\bar{\rho}_{s s}\right)$ decomposes over $\overline{\mathbb{F}}_{l}$ into the sum of irreducible characters $\chi_{1}+\ldots+\chi_{r}$. The operation of the standard Frobenius $F: x \mapsto x^{q}$, permutes the $\chi_{1}, \ldots, \chi_{r}$ (it follows from the Brauer-Nesbitt theorem, see Curtis and Reiner [5], Thm 30.16, that $\bar{\rho}_{s s}$ and $\bar{\rho}_{s s}^{F}$ have the same composition series because the characteristic polynomials of the Frobenius elements $\bar{\rho}_{s s}\left(\right.$ Frob $\left._{p}\right)$ remain unchanged). Now the theorem of Lang-Steinberg (see Carter [3], p. 32) implies that $\bar{\rho}_{s s}$ is equivalent to a representation $\hat{\rho}: \mathrm{G}_{\mathbb{Q}} \rightarrow G l\left(n, \mathbb{F}_{q}\right)$.

\section{Galois representations attached to Siegel modular forms}

The following theorem will be essential for our considerations:

Theorem 4. (Weissauer [24]) Suppose that $\Pi$ is a unitary cuspidal irreducible automorphic representation of $G S p(4, \mathbb{A})$ ( $\mathbb{A}$ denotes the ring of adeles of $\mathbb{Q}$ )

such that $\Pi_{\infty}$ belongs to the holomorphic discrete series of weight $\left(k_{1}, k_{2}\right)$. Set $w:=k_{1}+k_{2}-3$. Let $S$ denote the set of ramified places of the representation $\Pi$. Then there exists a number field $E$ such that for primes $p \notin S$ the local L-factor

$$
Q_{p}\left(p^{-s}\right)=L_{p}\left(\Pi_{p}, s-\frac{w}{2}\right), \quad L_{p}(X)^{-1} \in E[X],
$$

of the degree 4 spinor L-series (suitably normalized) has coefficients in E, and such that for any prime number $l$ and any extension $\lambda$ of $l$ to $E$ there exists a four dimensional semisimple Galois representation

$$
\rho_{\Pi, \lambda}: \mathrm{G}_{\mathbb{Q}} \rightarrow G l\left(4, \bar{E}_{\lambda}\right),
$$

which is unramified outside $S \cup\{l\}$ and for $p \notin S \cup\{l\}$ the following holds

$$
L_{p}\left(\Pi_{p}, s-\frac{w}{2}\right)=\operatorname{det}\left(1-\rho_{\Pi, \lambda}\left(\operatorname{Frob}_{p}\right) p^{-s}\right)^{-1} .
$$


First we want to reformulate the above theorem according to our situation:

Let $f$ be a classical Siegel modular form of even weight $k$ on the full Siegel modular group $S p(4, \mathbb{Z})$ which is a cusp form and a simultaneous eigenform for all Hecke operators $T(n), n \in \mathbb{N}$ (in the notation of Andrianov [1]).

One has a decomposition

$$
G S p(4, \mathbb{A})=G S p(4, \mathbb{Q}) G S p(4, \mathbb{R})^{+} \prod_{p<\infty} K_{p}
$$

where $G S p(4, \mathbb{R})^{+}$denotes the subgroup of elements of $G S p(4, \mathbb{R})$ having positive determinant and $K_{p}:=G S p\left(4, \mathbb{Z}_{p}\right)$, see, e.g., Weissauer [23]. One defines an automorphic form $\phi=\phi_{f}$ on $G S p(4, \mathbb{A})$ via

$$
\phi(g)=\phi\left(\gamma g_{\infty} k_{0}\right)=f\left(g_{\infty}\left(\mathrm{i} \cdot \operatorname{Id}_{2}\right)\right) \cdot \frac{\operatorname{det}\left(g_{\infty}\left(\mathrm{i} \cdot \mathrm{Id}_{2}\right)\right)^{k}}{\operatorname{det}\left(C \cdot \mathrm{i} \cdot \operatorname{Id}_{2}+D\right)^{k}}
$$

where

$$
g_{\infty}:=\left(\begin{array}{cc}
A & B \\
C & D
\end{array}\right) \in G S p(4, \mathbb{R})^{+}
$$

acts via linear fractional transformations, $\gamma \in G S p(4, \mathbb{Q})$ and $k_{0} \in \prod_{p<\infty} K_{p}$.

Then $\phi$ gives rise to a cuspidal irreducible automorphic representation $\Pi=\Pi_{f}$ of $G S p(4, \mathbb{A})$ whose ramification set is empty, see Asgari and Schmidt [2]. Moreover, $\Pi_{\infty}$ is in the holomorphic discrete series of weight $(k, k)$ (see $[2],[8]$ or $[25])$.

We will denote the corresponding Galois representations (given by the above theorem) by $\rho_{f, \lambda}$.

Let $\lambda_{n}$ denote the eigenvalue of $T(n)$ and let

$$
L_{f}(s):=\zeta(2 s-2 k+4) \sum_{n=1}^{\infty} \frac{\lambda_{n}}{n^{s}}
$$

be the spinor $L$-function. Then $L_{f}$ has an Euler product of the form

$$
L_{f}(s)=\prod_{p \in \mathbb{P}} Q_{p}\left(p^{-s}\right)^{-1},
$$

where $Q_{p}$ is the following polynomial:

$$
\begin{aligned}
& Q_{p}(X)=1-\lambda_{p} X+\left(\lambda_{p}^{2}-\lambda_{p^{2}}-p^{2 k-4}\right) X^{2}-\lambda_{p} p^{2 k-3} X^{3}+p^{4 k-6} X^{4} \\
= & \left(1-\left(\lambda_{p} / 2+\sqrt{d_{p}}\right) X+p^{2 k-3} X^{2}\right)\left(1-\left(\lambda_{p} / 2-\sqrt{d_{p}}\right) X+p^{2 k-3} X^{2}\right),
\end{aligned}
$$

with $d_{p}=-3 / 4 \lambda_{p}^{2}+\lambda_{p^{2}}+p^{2 k-4}+2 p^{2 k-3}$, see [18], p. 387 .

As a corollary to Theorem 4 we get:

Proposition 5. Let $f$ be a Siegel modular form of even weight $k$ on the full Siegel modular group $S p(4, \mathbb{Z})$ which is a cusp form and a simultaneous eigenform for all Hecke operators $T(n), n \in \mathbb{N}$. Let $E$ be the number field generated over 
$\mathbb{Q}$ by all the eigenvalues $\lambda_{n}, f \mid T(n)=\lambda_{n} f$. For any prime number $l$ and any extension $\lambda$ of $l$ to $E$ there exists a continuous Galois representation

$$
\rho_{f, \lambda}: \mathrm{G}_{\mathbb{Q}} \rightarrow G l\left(4, \bar{E}_{\lambda}\right)
$$

such that the following holds: The representation $\rho_{f, \lambda}$ is unramified outside $l$ and

$$
\operatorname{det}\left(\operatorname{Id}_{4}-X \cdot \rho_{f, \lambda}\left(\operatorname{Frob}_{p}\right)\right)=Q_{p}(X), \quad(p \neq l) .
$$

If $\rho_{f, \lambda}$ is absolutely irreducible, then the representation $\rho_{f, \lambda}$ is defined over $E_{\lambda}$.

Proof. All the statements except the last follow from the above discussion. The last statement can be found in Weissauer [24], App. D (alternatively one could use a result of Carayol [4]).

Remark 6. (i) If $\Pi_{f}$ has multiplicity one (that means $f$ is uniquely determined by its weight and its Hecke eigenvalues), the image of $\rho_{f, \lambda}$ is contained in a general symplectic group. See [24], Thm. IV.

ii) In the above proposition, it suffices to adjoin a Hecke eigenvalue of one element $T(p)$ (to obtain the field $E$ ) if the characteristic polynomial of $T(p)$ on the space spanned by the Galois translates of $f$ is irreducible over $\mathbb{Q}$. This follows from the existence of a basis of the space of Siegel modular forms of genus two of fixed weight whose elements have Fourier coefficients in $\mathbb{Q}$ (given by Igusa's theorem [9]).

\section{Computation of Hecke eigenvalues}

As in Skoruppa [18], [19], let

$$
\Upsilon=\Upsilon 28=\sum_{\substack{r, n, m \in \mathbb{Z} \\ r^{2}-4 m n \leq 0 \\ n, m \geq 0}} a(n, r, m) \cdot e^{n \cdot 2 \pi i \tau} \cdot e^{r \cdot 2 \pi i z} \cdot e^{m \cdot 2 \pi i \tau^{\prime}}, \tau, \tau^{\prime} \in \mathbb{H}, z \in \mathbb{C}
$$

be the Hecke eigenform of weight 28 which is orthogonal to the space of KlingenEisenstein series and the Maass-Spezialschar (an "interesting" formin the notation of Skoruppa [18]).

The Fourier coefficients depend only on the binary quadratic form $n X^{2}+$ $r X Y+m Y^{2}$, see Andrianov [1]. Skoruppa [19] computed the Fourier coefficients of $\Upsilon$ up to discriminant (of the corresponding quadratic form) -100 . Using this, one can compute the values $\lambda_{2}, \lambda_{4}, \lambda_{3}, \lambda_{9}$ and $\lambda_{5}, \lambda_{25}$, which determine the polynomials $Q_{2}, Q_{3}, Q_{5}$ of the previous section (Formula (1)).

As $\Upsilon$ is a Hecke eigenform we have the following identities, compare to [18], pp. 386-387: 


$$
\begin{aligned}
\lambda_{p} a(1,1,1)= & a(p, p, p)+p^{k-2}\left(1+\left(\frac{p}{3}\right)\right) a(1,1,1) \\
\lambda_{p} a(p, p, p)= & a\left(p^{2}, p^{2}, p^{2}\right)+p^{2 k-3} a(1,1,1)+p^{k-2} a\left(1, p, p^{2}\right)+ \\
& p^{k-2} \sum_{\nu \bmod p} a\left(1+\nu+\nu^{2}, p(1+2 \nu), p^{2}\right) \\
\lambda_{p^{2}} a(1,1,1)= & a\left(p^{2}, p^{2}, p^{2}\right)+p^{2 k-4}\left(\left(\frac{p}{3}\right)+\left(\frac{p^{2}}{3}\right)\right) a(1,1,1)+ \\
& p^{k-2} \sum_{\nu \bmod p: p \mid 1+\nu+\nu^{2}} a\left(1+\nu+\nu^{2}, p(1+2 \nu), p^{2}\right) .
\end{aligned}
$$

Moreover, since $a(1,3,9)=a(7,15,9)=a(1,1,7), a(1,5,25)=a(21,45,25)=$ $a(1,1,19)$ and $a(3,15,25)=a(7,25,25)=a(13,35,25)=a(3,3,7)$ we get

$$
\begin{array}{ll}
\lambda_{2}=\frac{a(2,2,2)}{a(1,1,1)}, & \lambda_{4}=\frac{a(4,4,4)}{a(1,1,1)} \\
\lambda_{3}=\frac{a(3,3,3)}{a(1,1,1)}+3^{(k-2)}, & \lambda_{9}=\frac{\lambda_{3} a(3,3,3)-3 \cdot 3^{(k-2)} a(1,1,7)}{a(1,1,1)}-3^{(2 k-3)}, \\
\lambda_{5}=\frac{a(5,5,5)}{a(1,1,1)}, & \lambda_{25}=\frac{\lambda_{5} a(5,5,5)-3 \cdot 5^{(k-2)} a(3,3,7)-3 \cdot 5^{(k-2)} a(1,1,19)}{a(1,1,1)} \\
-5^{(2 k-3)} .
\end{array}
$$

The following Fourier coefficients of $\Upsilon$ of can be found in [19]. Here $\alpha$ denotes some root of the polynomial $x^{3}-x^{2}-294086 x-59412960$ :

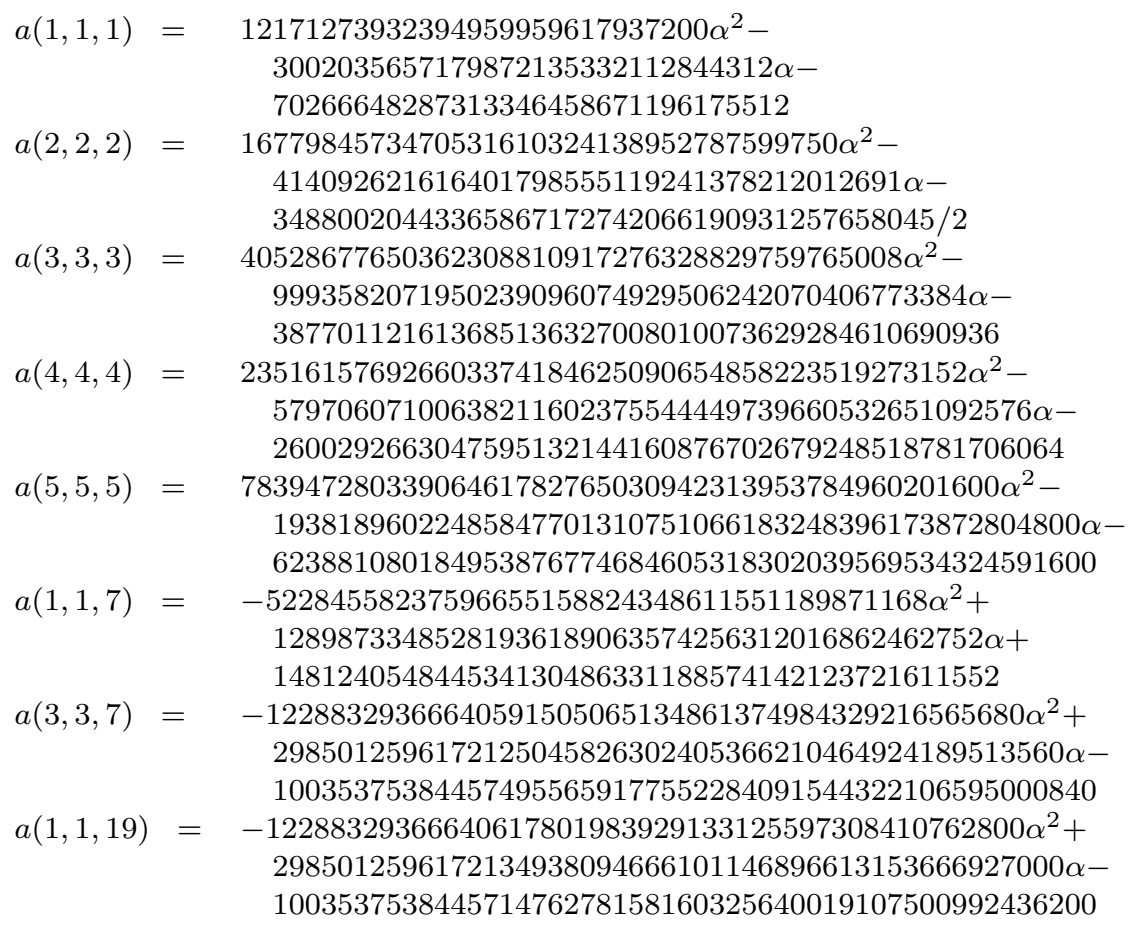

Table 4.1: Fourier coefficients of $\Upsilon 28$ 
Remark 7. The polynomial

$$
F(x):=x^{3}+137681664 x^{2}+4794374687293440 x+4100431555335920025600
$$

is the characteristic polynomial of the Hecke operator $T(2)$ on the space spanned by the Galois translates of $\Upsilon$. This space is exactly the complement of the space spanned by the Klingen-Eisenstein series and the Maass Spezialschar, see [18]. Thus the automorphic representation $\Pi_{\Upsilon}$ has multiplicity one.

\section{Some results on $G S p(4, q)$}

Consider $\beta_{1}, \beta_{2}, \eta \in \overline{\mathbb{F}}_{q}$, such that $\eta^{q}=\eta$ and such that the following equality holds:

$$
\left\{\beta_{1}, \eta \beta_{1}^{-1}, \beta_{2}, \eta \beta_{2}^{-1}\right\}=\left\{\beta_{1}^{q}, \eta \beta_{1}{ }^{-q}, \beta_{2}{ }^{q}, \eta \beta_{2}{ }^{-q}\right\} .
$$

The following table lists (up to conjugation) the tori in $G l(4, q)$ which are maximal with respect to the condition that their elements have a set of eigenvalues

$$
S(t)=\left\{\beta_{1}, \eta \beta_{1}^{-1}, \beta_{2}, \eta \beta_{2}^{-1}\right\} .
$$

\begin{tabular}{c|c|l} 
torus & order & eigenvalues \\
\hline$T_{0}$ & $(q-1)^{3}$ & $\left\{\beta_{1}, \eta \beta_{1}^{-1}, \beta_{2}, \eta \beta_{2}^{-1}\right\}, \beta_{i}^{q}=\beta_{i}$ \\
$T_{1}$ & $(q+1)^{2}(q-1)$ & $\left\{\beta_{1}, \eta \beta_{1}^{-1}, \beta_{2}, \eta \beta_{2}^{-1}\right\}, \beta_{i}^{q}=\eta \beta_{i}^{-1}$ \\
$T_{2}$ & $(q-1)^{2}(q+1)$ & $\left\{\beta_{1}, \eta \beta_{1}^{-1}, \beta_{2}, \eta \beta_{2}^{-1}\right\}, \beta_{1}^{q}=\beta_{1}, \beta_{2}^{q}=\eta \beta_{2}^{-1}$ \\
$T_{3}$ & $\left(q^{2}-1\right)(q-1)$ & $\left\{\beta_{1}, \beta_{1}^{q}, \eta \beta_{1}^{-1}, \eta \beta_{1}^{-q}\right\}, \beta_{1}^{q^{2}}=\beta_{1}$ \\
$T_{4}$ & $\left(q^{2}+1\right)(q-1)$ & $\left\{\beta_{1}, \beta_{1}^{q}, \eta \beta_{1}^{-1}, \eta \beta_{1}^{-q}\right\}, \beta_{1}^{q^{2}}=\eta \beta_{1}^{-1}$
\end{tabular}

Table 5.1

Note that these tori are (up to conjugation in $G l(4, q)$ ) maximal tori in $G S p(4, q)$ and $C O_{4}^{ \pm}(q)$, resp.. Thus if $S(t) \neq-S(t)$, we get $S\left(t^{\sigma}\right)=\eta^{-1} S(t), \sigma$ denoting the inverse-transpose map. If $S(t)=-S(t)$, we get $S\left(t^{\sigma}\right)=\eta^{-1} S(t)$ or $S\left(t^{\sigma}\right)=-\eta^{-1} S(t)$.

Remark 8. The numbers $r_{i}$ of irreducible factors (over $\mathbb{F}_{q}$ ) of the characteristic polynomial of a regular element of $T_{i}, i=0,1, \ldots, 4$, are $4,2,3,2,1$, respectively. Hence we can uniquely associate to a regular element $t$ (which has a set of eigenvalues $S(t)$ as above) a torus $T_{i}$, except in the case $t \sim$ $\operatorname{diag}\left(\beta,-\eta \beta^{-1}, \eta \beta^{-1},-\beta\right), \beta^{q}=-\eta \beta^{-1}$.

For $\rho_{f, \lambda}: \mathrm{G}_{\mathbb{Q}} \rightarrow G l\left(4, \bar{E}_{\lambda}\right)$ as in Prop. 5 , let $\mathcal{G}:=\operatorname{im}\left(\rho_{f, \lambda}\right)$. Then, let $\overline{\mathcal{G}}$ be the Zariski closure of $\mathcal{G}$ in $G l\left(4, \bar{E}_{\lambda}\right), \overline{\mathcal{G}}^{0}$ be the identity component of $\overline{\mathcal{G}}$ and $\mathcal{G}^{0}:=\overline{\mathcal{G}}^{0} \cap \mathcal{G}$. Let

$$
\hat{\rho}_{f, \lambda}: \mathrm{G}_{\mathbb{Q}} \rightarrow G l(4, q), \mathbb{F}_{q}=k_{E_{\lambda}},
$$

be as in Lemma $3, G$ be the image of $\hat{\rho}_{f, \lambda}$ and $G^{0}=\hat{\rho}_{f, \lambda}\left(\rho_{f, \lambda}^{-1}\left(\mathcal{G}^{0}\right)\right)$.

From the list of Taylor [20], bottom of page 298, we deduce that $G^{0}$ is contained in one of the groups listed below: 


\begin{tabular}{l|l|l|} 
overgroups of $G^{0}$ & tori & $\left|\overline{\mathcal{G}}^{0}\right| \overline{\mathcal{G}}^{0} \mid$ \\
\hline$C O_{4}^{+}(q)=G l(2, q) \circ G l_{2}(q)=\{X \otimes Y \mid X, Y \in G l(2, q)\}$ & $T_{0}, T_{1}, T_{3}$ & $\leq 2$ \\
$C O_{4}^{-}(q) \cong G l\left(2, q^{2}\right)=\left\{X \otimes X^{F_{q}} \mid X \in G l\left(2, q^{2}\right)\right\}$ & $T_{2}, T_{4}$ & $\leq 2$ \\
& & \\
\hline & & \\
$G S p(4, q)$ & & \\
$G l(2, q)=\{\operatorname{diag}(X, X) \mid X \in G l(2, q)\}$ & $T_{1}, T_{2}$ & $\leq 2$ \\
$G l(2, q)=\left\{\operatorname{diag}\left(Y, X, \operatorname{det}(X) Y^{-1}\right) \mid Y \in G l_{1}(q), X \in G l(2, q)\right\}$ & $T_{0}, T_{2}$ & $\leq 2$ \\
$G l(2, q)=\left\{\operatorname{diag}\left(Y, X, \operatorname{det}(X) Y^{-1}\right) \mid Y \in G U_{1}(q), X \in G l(2, q)\right\}$ & $T_{1}, T_{2}$ & $\leq 2$ \\
$G l(2, q) \times G l_{1}(q)=\left\{\operatorname{diag}\left(X, \eta X^{\sigma}\right) \mid X \in G l(2, q), \eta \in \mathbb{F} q\right\}$ & $T_{0}, T_{3}$ & $\leq 2$ \\
$G U_{2}(q) \times G l_{1}(q)=\left\{\operatorname{diag}\left(X, \eta X^{\sigma}\right) \mid X \in G U_{2}(q), \eta \in \mathbb{F} q\right\}$ & $T_{1}, T_{3}$ & $\leq 2$ \\
$\{(X, Y) \mid X, Y \in G l(2, q), \operatorname{det}(X)=\operatorname{det}(Y)\}$ & $T_{0}, T_{1}, T_{2}$ & $\leq 2$ \\
$\left\{\left(X, X F_{q}\right) \mid X \in G l\left(2, q^{2}\right), \operatorname{det}(X) \in \mathbb{F} q\right\}$ & $T_{3}, T_{4}$ & $\leq 2$ \\
$T_{i}, i=0, \ldots, 4$ & & $\leq 8$
\end{tabular}

Table 5.2

Remark 9. Let $s \in N_{G l(4, q)}(H) \backslash H$, where $H \neq G S p(4, q)$ is one of the groups of the above list. Then $s$ has a pair of eigenvalues $\left(\beta_{1},-\beta_{1}\right)$.

Proof. First, let $s^{2} \in C O_{4}^{ \pm}(q)$ and $s \notin C O_{4}^{ \pm}(q)$. Then $s \sim \operatorname{diag}\left(\beta_{1}, \beta_{2}, \eta \beta_{2}{ }^{-1}\right.$, $\left.-\beta_{1}\right), \beta_{1}^{2}=\eta \in \mathbb{F}_{q}, \beta_{2} \in \mathbb{F}_{q^{2}}$ (see [13]). In any other case $N_{G l(4, q)}(H)$ is an imprimitive group, where $s$ permutes 2 or 4 blocks. Hence $s^{2}$ is not a regular element and the claim follows.

Proposition 10. Let $l$ be an odd prime such that the residue field $\mathbb{F}_{q}=k_{E_{\lambda}}$ is not a prime field and let $G=\operatorname{im}\left(\hat{\rho}_{f, \lambda}\right)$. Further assume that $\mathbb{F}_{q}$ is generated over $\mathbb{F}_{l}$ by the reduced coefficients of the characteristic polynomials of the Frobenius elements. If $G$ contains elements $t_{0}, t_{4}$, resp. $t_{1}, t_{4}$, resp. $t_{2}, t_{3}$, where $t_{i}$ is contained up to conjugation in the maximal torus $T_{i}\left(T_{i}\right.$ as in Table 5.1) and the element $t_{i}^{2}$ is regular, then $S p(4, q) \leq G$.

Proof. We exclude all the possible overgroups $H \neq G S p(4, q)$ of $G^{0}(H$ as in Table 5.2) by considering the maximal tori contained in $H$, together with Remark 9 .

To treat the group $G S p(4, q)$ note that the above list contains all maximal subgroups of $G S p(4, q)$ with the possible exception $G S p\left(4, q_{0}\right), q=q_{0}^{r}, r$ a prime, and maximal subgroups of type $S$ (in the notation of Kleidman and Liebeck [10]).

The maximal subgroups of type $S$ are excluded by noting that the condition that $q$ is not a prime implies, that one has no maximal subgroups of type $S$, compare to Mitchell [12].

To exclude the case $G S p\left(4, q_{0}\right)$ note that we can assume by Formula (1) and Proposition 5 that $G$ is contained in the group $\mathbb{F}_{l^{2}} \cdot S p\left(4, q_{0}\right)$. This implies that the compositum of $\mathbb{F}_{l^{2}}$ and $\mathbb{F}_{q_{0}}$ is $\mathbb{F}_{q}$. So we can assume that $\left[\mathbb{F}_{q}: \mathbb{F}_{q_{0}}\right]=2$. Now observe that in this case a regular element contained in $T_{2}$, resp. $T_{4}$, is not contained in the group $\mathbb{F}_{l^{2}} \cdot S p\left(4, q_{0}\right)$. 
Proposition 11. Let $G$ be as above. Suppose $S p(4, q) \leq G$. Then $G / Z(G)=$ $P S p(4, q)$ if $m$ is even and $G / Z(G)=P G S p(4, q)$ if $m$ is odd.

Proof. By assumption we get $G / Z(G)=P S p(4, q)$ or $G / Z(G)=P G S p(4, q)$. Let $t_{p}=\hat{\rho}_{f, \lambda}\left(\right.$ Frob $\left._{p}\right)$. Since $G=\left\langle t_{p} \mid p \in \mathbb{P}\right\rangle \leq G S p(4, q)$, we find by Prop. 5 that $t_{p}^{\sigma}=p^{2 k-3} t_{p}$. Thus if $m$ is even we get $(q-1)_{2}>(l-1)_{2}$. Hence $G \leq$ $Z(G l(4, q)) S p(4, q)$. Now let $m$ be odd. Then $p^{2 k-3}$ is a non square in $\mathbb{F}_{q}$ if $p$ is a non square in $\mathbb{F}_{l}$. Assume $\left(\frac{p}{l}\right)=1$ for all $p \in \mathbb{P}$. Then $\left(\frac{n}{l}\right)=1$ for $1 \leq n<l$ odd. Hence $\left(\frac{n}{l}\right)=-1$ for $1 \leq n<l$ even. Since $\left(\frac{4}{l}\right)=1$ we have $4>l$, hence $l=3$. On the other hand we have $\left(\frac{5}{3}\right)=-1$, a contradiction.

\section{The resulting Galois realizations}

Definition 12. A profinite group $G$ occurs as Galois group over $\mathbb{Q}$ if there exists a continuous homomorphism of $\mathrm{G}_{\mathbb{Q}}$ onto $G$.

Let $f$ be a Siegel modular form of weight $k$ as in Section 3 , and let $E, \lambda$, and

$$
\rho_{f, \lambda}: \mathrm{G}_{\mathbb{Q}} \rightarrow \operatorname{Gl}\left(4, \bar{E}_{\lambda}\right)
$$

be as in Prop. 5. If $\Pi_{f}$ has multiplicity one, the groups

$$
\mathcal{A}_{\lambda}^{k}:=\left\{g \in G S p\left(4, O_{E_{\lambda}}\right) \mid \operatorname{det}(g) \in\left(\mathbb{Z}_{l}^{\times}\right)^{4 k-6}\right\}
$$

are natural candidates for the images of $\rho_{f, \lambda}$ by Formula (1) and Remark 6 . In the following, let $\Upsilon=\Upsilon 28$ be the Hecke eigenform of Section 4. Then $E:=\mathbb{Q}(\alpha)$, where $\alpha$ is a root of $x^{3}-x^{2}-294086 x-59412960$ (using Remark 6 ii) and the irreducibility of $F(x)$ over $\mathbb{Q}$, where $F(x)$ is as in Remark 7).

Theorem 13. i) For infinitely many primes numbers $l$ (especially $l=53$ ) there exists an extension $\lambda$ of $l$ to $E$ of inertia degree 3 (i.e. $k_{E_{\lambda}} \simeq \mathbb{F}_{l^{3}}$ ) such that

$$
\rho_{\Upsilon, \lambda}\left(G_{\mathbb{Q}}\right)=\mathcal{A}_{\lambda}^{28} \text {. }
$$

In particular, the factor groups $P G S p\left(4, O_{E_{\lambda}}\right)$ and $P G S p\left(4, l^{3}\right)$ of $\mathcal{A}_{\lambda}^{28}$ occur as Galois groups over $\mathbb{Q}$. The corresponding Galois extensions of $\mathbb{Q}$ are unramified outside $l$.

ii) For infinitely many primes numbers $l^{\prime}$ (especially $\left.l^{\prime}=19\right)$ there exists an extension $\lambda^{\prime}$ of $l^{\prime}$ to $E$ of inertia degree 2 such that

$$
\rho_{\Upsilon, \lambda^{\prime}}\left(G_{\mathbb{Q}}\right)=\mathcal{A}_{\lambda^{\prime}}^{28}
$$

In particular, the factor groups $P S p\left(4, O_{E_{\lambda^{\prime}}}\right)$ and $P S p\left(4, l^{\prime 2}\right)$ of $\mathcal{A}_{\lambda^{\prime}}^{28}$ occur as Galois groups over $\mathbb{Q}$. The corresponding Galois extensions of $\mathbb{Q}$ are unramified outside $l^{\prime}$.

Proof. (i) Let first $l=53$. Since 53 does not divide the discriminant of $E(=$ $5 \cdot 13 \cdot 73693 \cdot 1418741)$ it follows that 53 is unramified. Let $\lambda$ be an extension of 53 to $E$. Then, since $F(x)$ modulo $\lambda$ is irreducible we have $k_{E_{\lambda}} \simeq \mathbb{F}_{53^{3}}$.

Let $t_{p}$ denote the image of a Frobenius element Frob $_{p}$ under the image of $\hat{\rho}_{\Upsilon, \lambda}$, where $\hat{\rho}_{\Upsilon, \lambda}$ is as in Lemma 3. By Lemma 3 we get that $t_{p}$ is contained in $G l\left(4,53^{3}\right)$. (By the definition of the polynomials $Q_{p}, E$ also coincides with 
the number field which is generated over $\mathbb{Q}$ by the coefficients of the characteristic polynomials of the Frobenii.) Using the results of Section 4 together with Formula (1) of Section 3 one sees that

$$
\begin{aligned}
& Q_{2}(x) \equiv 4\left(x^{2}+\left(45 \alpha^{2}+19 \alpha+33\right) x+28 \alpha^{2}+47 \alpha+39\right) \\
&\left(x^{2}+\left(48 \alpha^{2}+8 \alpha+46\right) x+17 \alpha^{2}+15 \alpha\right) \bmod \lambda \\
& Q_{3}(x) \equiv \begin{array}{l}
9\left(x+39 \alpha^{2}+15 \alpha+27\right)\left(x+16 \alpha^{2}+28 \alpha+9\right) \\
\left(x+5 \alpha^{2}+5 \alpha+28\right)\left(x+46 \alpha^{2}+18 \alpha+39\right) \bmod \lambda
\end{array} \\
& Q_{5}(x) \equiv \begin{array}{l}
1+25\left(27 \alpha^{2}+51 \alpha+52\right) x+25\left(47 \alpha^{2}+24+26 \alpha\right) x^{2}+ \\
25\left(29 \alpha^{2}+43 \alpha+48\right) x^{3}+25 x^{4} \bmod \lambda .
\end{array}
\end{aligned}
$$

From the list of polynomials $Q_{2}, Q_{3}, Q_{5} \bmod \lambda$, together with Proposition 5 and Lemma 3, we deduce that $t_{2} \in T_{3}, t_{3} \in T_{0}, t_{5} \in T_{4}$ and $t_{3}^{2}, t_{5}^{2}$ are regular elements. Thus by Proposition 10 the group $H:=\left\langle t_{2}, t_{3}, t_{5}\right\rangle$ contains the group $S p\left(4,53^{3}\right)$; in particular, $H$ is absolutely irreducible. Using the list of the possible overgroups in Section 5 (or by multiplicity one, see Remarks 6 i) and 7) we find that $H$ is contained in the group $G S p\left(4,53^{3}\right)$.

Since $H$ is absolutely irreducible, it follows by definition that $\hat{\rho}_{\Upsilon, \lambda}$ is absolutely irreducible. It follows from the construction of $\hat{\rho}_{\Upsilon, \lambda}$ that also $\rho_{\Upsilon, \lambda}$ is absolutely irreducible. Thus the representation $\rho_{\Upsilon, \lambda}$ is defined over $E$ (see Prop. 5). Since $H / Z(H)$ contains $P S p\left(4, l^{3}\right)$ it follows $\operatorname{Im}\left(\rho_{\Upsilon, \lambda}\right)=\mathcal{A}_{\lambda}^{28}$, using Lemma 2 and $\operatorname{det}\left(\operatorname{Frob}_{p}\right)=p^{4 k-6}$. The groups $P G S p\left(4, O_{E_{\lambda}}\right)$ and $P G S p\left(4, l^{3}\right)$ are factor groups of $\mathcal{A}_{\lambda}^{28}$ by Prop. 11 and the corresponding Galois extensions are unramified outside $l$ by Prop. 5 .

We use Cebotarev's density theorem in order to show that analogous statements hold for infinitely many primes $l$ : Let $E^{\prime}$ be the Galois closure of $E$ and

$$
\tilde{Q}:=\prod_{i=2,3,5} \cdot \prod_{\sigma \in \operatorname{Gal}\left(E^{\prime}: \mathbb{Q}\right)} Q_{i}^{\sigma} \in \mathbb{Q}[x] .
$$

Looking at the case $l=53$ one sees (by applying Cebotarev's density theorem to $\tilde{Q})$ that there are infinitely many primes $l$ of inertia degree 3 in $E$ such that the polynomials $Q_{i}, i=2,3,5$, decompose modulo $l$ as in the 53 case above. Then continue as in the 53 case.

ii) Let first $l^{\prime}=19$ : We have

$$
F(x) \equiv(x+18)\left(x^{2}+8 x+17\right) \bmod l^{\prime} .
$$

Let $\lambda^{\prime}$ be the extension of $l^{\prime}$ to $E$ of inertia degree 2 and let $\alpha \in k_{E_{\lambda^{\prime}}}=\mathbb{F}_{19^{2}}$ be a root of $x^{2}+7 x+14$. Then

$$
\begin{aligned}
& Q_{2}(x) \equiv 1+5(17 \alpha+14) x+5(6+13 \alpha) x^{2}+5(18 \alpha+7) x^{3}+5 x^{4} \quad \bmod \lambda^{\prime} \\
& Q_{3}(x) \equiv 17\left(x^{2}+(\alpha+8) x+3\right)\left(x^{2}+(16 \alpha+4) x+3\right) \bmod \lambda^{\prime} \\
& Q_{5}(x) \equiv 16(x+9 \alpha+12)(x+1)(x+5)(x+14 \alpha+16) \quad \bmod \lambda^{\prime}
\end{aligned}
$$

Thus $t_{2} \in T_{4}, t_{3} \in T_{1}, t_{5} \in T_{0}$. The claim on $l^{\prime}=19$ as well as the claim on the infinitely many other $l^{\prime}$ 's follow now analogously as in i). 
Remark 14. i) The Galois representations attached to Hecke eigenforms in the Maass Spezialschar are related to two dimensional Galois representations; in particular, they are always reducible, see [24], Thm. II. The Fourier coefficients of the non-Maass, non-Eisenstein, Hecke eigenforms of smaller weights lie completely in $\mathbb{Q}$ (see [18]) and thus cannot yield anything new from the inverse Galois theoretic viewpoint (see Malle and Matzat [11], Serre [17] and Völklein [22] for an introduction to the inverse Galois problem), since it is well known that the groups $P G S p(2 n, l)$ occur as Galois groups over $\mathbb{Q}$ (e.g. use [11], Thm. I.8.6, together with [13], Satz 6.1, 7.1, as in [11], Ex. I.8.2.).

ii) One can realize symplectic groups $P S p\left(n, l^{m}\right), l$ odd, for a fixed $m \in \mathbb{N}$ (regularly) as Galois groups over $\mathbb{Q}$ if $n>l^{m}$ (see [7], extending results of Thompson and Völklein [21]). It is in general very hard to realize symplectic groups regularly as Galois groups over $\mathbb{Q}$ if $n$ lies below this bound and $m>1$, because the Hurwitz spaces which parametrize the corresponding families of covers tend to have "few" rational points. This is partly because these Hurwitz spaces are often varieties of general type, partly because of the action of the absolute Galois group on the conjugacy classes via the cyclotomic character (see $[11]$ and [22] for this).

\section{References}

[1] Andrianov, A.N., Quadratic forms and Hecke operators, Berlin: Springer Verlag (1987).

[2] Asgari, M., Schmidt, R., Siegel modular forms and representations, Manuscripta Math. 104 (2001), No. 2, 173-200.

[3] Carter, R., Finite Groups of Lie Type: Conjugacy classes and complex characters, New York: Wiley (1985).

[4] Carayol, H., Formes modulaires et représentations Galoisiennes à valeurs dans un anneau local complet, in: $p$-adic Monodromy and the Birch-Swinnerton-Dyer Conjecture (Mazur and Stevens eds.), Contemp. Math. 165 (1994), Amer. Math. Soc., 193-236.

[5] Curtis, C.W., Reiner, I., Representation Theory of Finite Groups and Associative Algebras. New York: Wiley (1962).

[6] Deligne, P., Formes modulaires et représentations l-adiques, Seminaires Bourbaki 355, Fevrier 1969, Springer LNM 179 (1971).

[7] Dettweiler, M., Reiter, S., An algorithm of Katz and its application to the inverse Galois problem, Journal of Symb. Comp. 30 (2000), No. 6, 761-798.

[8] Faltings, G., Chai, C.-L., Degeneration of Abelian Varieties, Berlin, Heidelberg: Springer Verlag (1990).

[9] Igusa, J.-I., On Siegel modular forms of genus two, Amer. J. Math. 84 (1962), 175-200.

[10] Kleidman, P., Liebeck, M., The subgroup structure of the finite classical groups. Cambridge University Press (1990).

[11] Malle, G., Matzat, B.H., Inverse Galois theory, Berlin, Heidelberg: Springer Monographs in Mathematics (1999).

[12] Mitchell, H.H., The subgroups of the quaternary abelian linear group, Trans. AMS 15 (1914), 379-396.

[13] Reiter, S., Galoisrealisierungen klassischer Gruppen, J. Reine Angew. Math. 511 (1999), 193-236.

[14] Reverter, A., Vila, N., Some projective linear groups over finite fields as Galois groups over $\mathbb{Q}$., Contemp. Math., Ed. Fried, M. et al. 186 (1995), 51-63.

[15] Ribet, K.A., On l-adic representations attached to modular forms, Invent. Math. 28 (1975), 245-275. 
[16] Serre, J.-P., Abelian l-adic Representations and Elliptic Curves, New York: Addison Wesley (1989).

[17] Serre, J.-P., Topics in Galois Theory, Boston: Jones and Bartlett (1992).

[18] Skoruppa, N.-P., Computations of Siegel modular forms of genus two, Mathematics of Computation 58 (1992), 381-398.

[19] Skoruppa, N.-P., Siegel Modular Eigenforms of Even Weight on the Full Siegel Modular Group of Genus 2, (1999). Available under http://thor.math.u-bordeaux.fr/ modi/.

[20] Taylor, R., On the l-adic cohomology of Siegel threefolds, Invent. Math. 114 (1993), 289-310.

[21] Thompson, J., Völklein, H., Symplectic groups as Galois groups, J. Group Theory 1 (1998), 1-58.

[22] Völklein, H., Groups as Galois groups. New York: Cambridge Studies in Advanced Mathematics 53 (1996).

[23] Weissauer, R., Differentialformen zu Untergruppen der Siegelschen Modulgruppe zweiten Grades, J. Reine Angew. Math. 391 (1988), 100-156.

[24] Weissauer, R., Four dimensional Galois representations, Preprint (1993, 2000).

[25] Weissauer, R., An application of the hard Lefschetz theorem, Preprint (1993).

IWR, Universität Heidelberg, Im Neuenheimer Feld 368, 69120 Heidelberg, GerMANY.

E-mail address: michael.dettweiler@iwr.uni-heidelberg.de.

Institut für Mathematik, Rudower Chaussee 25, Humboldt-Universität Zu Berlin, 10099 Berlin, Germany.

E-mail address: kuehn@mathematik.hu-berlin.de

IWR, Universität Heidelberg, Im Neuenheimer Feld 368, 69120 Heidelberg, GerMANY.

E-mail address: reiter@iwr.uni-heidelberg.de 